Bull. Mater. Sci. Vol. 17, No. 6, November 1994, pp. 1039-1047. (C) Printed in India.

\title{
Growth, characterization and electrical anisotropy in GaTe-a natural semiconducting superlattice
}

\author{
$S$ PAL and D N BOSE \\ Materials Science Centre, Indian Institute of Technology, Kharagpur 721 302, India
}

\begin{abstract}
GaTe is a III-VI semiconductor which has layered structure with large anisotropy in electrical properties. Growth of single crystals by the Bridgman technique permitted the measurement of thermoelectric power in orthogonal directions from which the anisotropy of hole effective masses were determined for the first time. From resistivity and Hall effect measurements the carrier activation energies and scattering mechanisms between $10-300^{\circ} \mathrm{K}$ were found.

Study of the temperature dependence of conductivity revealed a variety of conduction mechanisms including weak localization below $20^{\circ} \mathrm{K}$, hopping conduction between $20-50$ $\mathrm{K}$ and band conduction in and across the layer planes at $T>70 \mathrm{~K}$. Weak localization was confirmed through observation of negative magnetoresistance. The $I-V$ characteristics showed quantized behaviour due to tunneling across potential barriers, which may be due to stacking faults between layer planes as observed by TEM studies.
\end{abstract}

Keywords. GaTe; III-VI semiconductor; electrical anisotropy.

\section{Introduction}

GaTe crystallizes in the monoclinic system (space group $C_{2}^{2}$ ). According to the Mooser-Pearson criterion it is a III-VI semiconductor having one cation-cation (M-M) bond and forms $\mathrm{X}-\mathrm{M}-\mathrm{M}-\mathrm{X}$ chains. There is strong covalent bonding within the layer planes with weak van der Waals bonding between them resulting in easy cleavage. Due to difficulties in crystal growth only the in-plane properties have been reported by Fischer and Brebner (1962), Manfredotti et al (1975) and Segura et al (1989). The subject has been reviewed by Fivaz and Schmid (1976).

In the present work high quality crystals of InTe and GaTe were grown by the Bridgman technique using very slow growth rates after synthesis from the elements. The crystals were characterized by Laue back reflection and electron diffraction for structure followed by energy dispersive $\mathrm{X}$-ray analysis (EDAX) and X-ray photoelectron spectroscopy (XPS) to establish stoichiometry. Impurity analysis was carried out using inductively coupled plasma emission (ICP). The optical band-gap at $300 \mathrm{~K}$ was found to be $1.66 \mathrm{eV}$ and direct in nature. Detailed studies of the electrical transport properties between $10-350^{\circ} \mathrm{K}$ were thereafter carried out as will be described in this paper. The study of deep levels in orthogonal directions in GaTe has already been reported by Pal et al (1994). A new semiconducting ferroelectric has also been found in the related $\mathrm{Ge}_{1-x} \mathrm{Ga}_{x} \mathrm{Te}$ system by Bose and Pal (1994).

\section{Crystal growth}

$\mathrm{Ga}$ and $\mathrm{Te}$ of $6 \mathrm{Ns}$ purity were used for synthesis of the compound in sealed quartz 
tubes using prolonged and continuous rotation for homogenization. The material was then placed in a conically-tipped ampule in a 3-zone vertical furnace and heated to temperatures well above the melting point of $1097 \mathrm{~K}$. The charge was lowered at $1-1.2 \mathrm{~mm} / \mathrm{h}$. A short post-growth anneal at $100-120^{\circ} \mathrm{C}$ was carried out to give the desired monoclinic phase. The resulting ingots were $1.25 \mathrm{~cm}$ in diameter and $2.5 \mathrm{~cm}$ in length ( $\mathrm{Pal}$ et al 1994) and could be cleaved easily parallel to the layer planes.

\section{Characterization}

X-ray powder diffraction studies were carried out which confirmed the monoclinic structure of GaTe with unit cell parameters $a=17.32 \mathrm{~A}, b=4.05 \mathrm{~A}$ and $c=10.59$ $A$ with $\beta=104.4^{\circ}$. This corresponded to $\mathrm{Ga}-\mathrm{Ga}$ and $\mathrm{Ga}-\mathrm{Te}$ bond lengths of 2.473 and $2.638 \mathrm{~A}$ respectively and Te-Te distance of $4.096 \mathrm{~A}$. Laue back-reflection showed that the (001) direction was along the layer plane. Electron diffraction studies using a Philips transmission electron microscope (TEM) at $100 \mathrm{keV}$ were also carried out. The diffraction pattern showed the expected 2-fold symmetry and confirmed the values of the lattice parameters. Electron micrographs showed the presence of dislocations in the basal plane and ribbons of stacking faults similar to those in GaSe.

The Ga: Te ratio determined by EDAX and XPS are compared in table 1 which shows reasonable agreement between the bulk and surface.

ICP analysis was conducted on the elements as well as the compound after crystal growth. The principal impurities at trace levels were found to be $\mathrm{Pb}(0.67$ $\mathrm{ppm}), \mathrm{Sn}(0.56 \mathrm{ppm}), \mathrm{Fe}(0.34 \mathrm{ppm})$ and $\mathrm{Mg}(0.26 \mathrm{ppm})$, the first originating from $\mathrm{Ga}$ and the others from $\mathrm{Te}$.

\section{Optical absorption}

Thin platelets were cleaved from the crystals and polished to mirror finish. The optical absorption was studied perpendicular to the layer plane at $300 \mathrm{~K}$ using a UV 365 Shimazu spectrophotometer. The absorption plotted as $(\alpha h v)^{2}$ vs $h v$ gave a straight line indicating a direct gap of $1.66 \pm 0.002 \mathrm{eV}$ in good agreement with a value of $1.663 \mathrm{eV}$ reported by Brebner and Fischer (1962).

\section{Thermoelectric power (TEP)}

TEP was measured between 140 and $300 \mathrm{~K}$ maintaining a constant temperature

Table 1. Compositional analysis of GaTe single crystals.

\begin{tabular}{lll}
\hline & Ga (at \%) & Te (at \%) \\
\hline EDAX & $49.2-49.3$ & $50.8-50.7$ \\
XPS & $49.1-49.2$ & $50.9-50.8$ \\
\hline
\end{tabular}


difference of $7-8^{\circ} \mathrm{C}$ between the ends of the samples aligned along or perpendicular to the layer planes. The sign of the TEP was always positive indicating hole conduction. The nature of variation for the two cases is shown in figure 1 . At $300 \mathrm{~K}$ the TEP in the layer plane was $873 \mu \mathrm{V} / \mathrm{K}$ which increased to $1200 \mu \mathrm{V} / \mathrm{K}$ at $140 \mathrm{~K}$. In the perpendicular direction at $300 \mathrm{~K}$ TEP was $1233 \mu \mathrm{V} / \mathrm{K}$, went through a minimum and thereafter increased to $1550 \mu \mathrm{V} / \mathrm{K}$ at $140 \mathrm{~K}$. The activation energies in two temperature regions given in table 2 were in good agreement with the results of Hall effect measurements. The decrease in TEP with increase in temperature is essentially due to the increase in hole concentration i.e. the Fermi level moves towards the valence band in this temperature range and hence $E_{\mathrm{F}}-E_{\mathrm{v}}$ in (1) decreases.

TEP is related to the Fermi level in a p-type semiconductor by

$$
S=+\frac{k}{e}\left[\frac{E_{\mathrm{F}}-E_{\mathrm{v}}}{k T}+A+\frac{5}{2}\right],
$$

where $A$ is a constant whose value depends on the scattering mechanism being $3 / 2,-1 / 2$ or $+1 / 2$ for impurity, acoustical phonon or optical phonon scattering respectively. For GaTe along the layer planes between $140-300 \mathrm{~K}, A=+1 / 2$

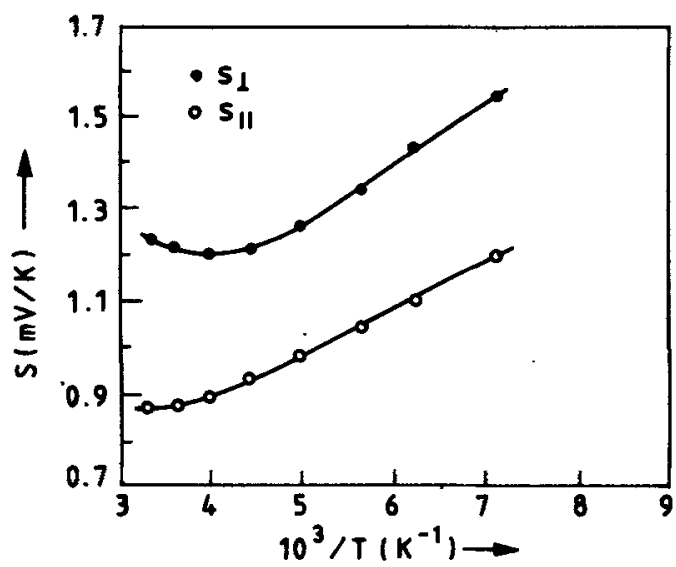

Figure 1. Temperature dependence of thermoelcctric power $S$ for $p$-GaTe parallel and perpendicular to layer plane.

Table 2. Activation energies and hole effective masses in GaTe from TEP studies.

\begin{tabular}{lcccc}
\hline Orientation & $\begin{array}{c}E_{\mathrm{a}}(\mathrm{meV}) \\
(80-100 \mathrm{~K})\end{array}$ & $\begin{array}{c}E_{\mathrm{a}}(\mathrm{meV}) \\
(100-250 \mathrm{~K})\end{array}$ & $m_{\mathrm{h}}^{*}$ & $A$ \\
\hline Parallel to layer plane & 22.5 & 73.6 & $\begin{array}{c}0.465 \mathrm{~m}_{\mathrm{0}} \\
( \pm 0.01)\end{array}$ & +0.5 \\
Perpendicular to layer plane & 39.7 & 111.0 & $\begin{array}{c}0.995 \mathrm{~m}_{\mathrm{0}} \\
( \pm 0.01)\end{array}$ & +4.5 \\
\hline
\end{tabular}


indicating optical phonon scattering whereas in the perpendicular direction $A=4.5$ in the same temperature range. This cannot be explained by any of the above scattering mechanisms and may be due to the existence of planar defects between the layers. The hole effective masses were calculated from the values of $N_{\mathrm{v}}$ in the two directions and were found to be $0.465 m_{0}$ and $0.995 m_{0}$ respectively as given in table 2.

\section{Resistivity and Hall effect}

The resistivity of samples were measured both along and perpendicular to the layer planes by the van der Pauw method. Ohmic contacts were made by indium evaporation followed by annealing at $200^{\circ} \mathrm{C}$ in Argon atmosphere for 5 min. Hall effect measurements were carried out between $77-300 \mathrm{~K}$ at magnetic fields up to $0.5 \mathrm{~T}$. Arrhenius plots of $\ln \sigma$ and $\ln \sigma$ vs $1 / T$ are shown in figure 2 . Two sets of activation energies were obtained from the graphs in each direction between 80 and $100 \mathrm{~K}$ and 100 and $250 \mathrm{~K}$. The values along the layer planes were $0.023 \mathrm{eV}$ and $0.074 \mathrm{eV}$ respectively lower than the corresponding values $0.040 \mathrm{eV}$ and $0.110 \mathrm{eV}$ in the perpendicular direction. In general $\sigma_{\sharp} \gg \sigma_{\perp}$ as expected for layered compounds the ratio known as the anisotropy factor being given by

$$
\sigma_{\|} / \sigma_{\perp}=A \exp (\Delta E / k T)=m_{\perp}^{*} / m_{\|}^{*} \exp (\Delta E / k T),
$$

where $m_{\perp}^{*}$ and $m_{\|}^{*}$ are the effective masses. The value of $\Delta E$ was found experimentally to be $0.04 \mathrm{eV}$ between 182 and $30 \mathrm{~K}$ and shows the effect of defects on $\sigma_{\perp}$.

Figure 3 shows the variation of hole mobility with temperature in the two directions between 80 and $300 \mathrm{~K}$ with $p=(2.75-6.5) \times 10^{15} / \mathrm{cm}^{3}$. At $300 \mathrm{~K}$ $\mu_{11}=25-40 \mathrm{~cm}^{2} / \mathrm{Vsec}$ and $\mu_{\perp}=10-15 \mathrm{~cm}^{2} / \mathrm{Vsec}$ while near $80 \mathrm{~K}$ these values increased to $450-500 \mathrm{~cm}^{2} / \mathrm{Vsec}$ and $100-110 \mathrm{~cm}^{2} / \mathrm{Vsec}$ respectively. These are much

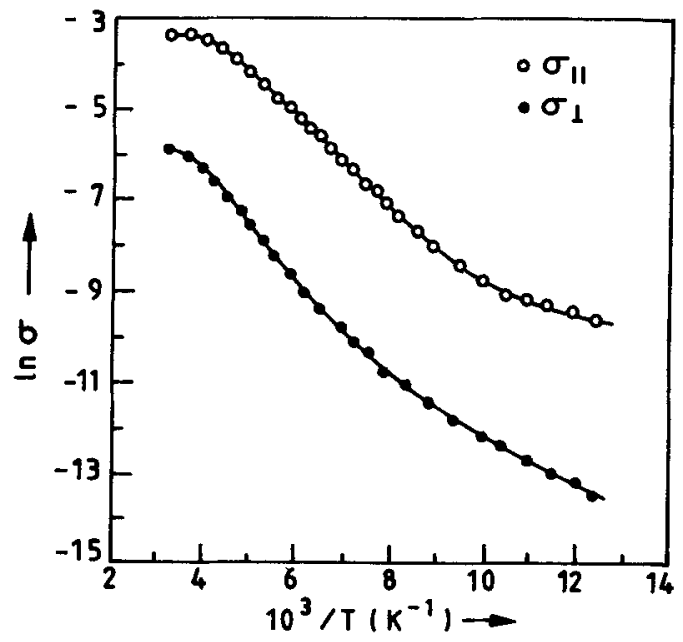

Figure 2. Temperature dependence of conductivity in $p$-GaTe measured parallel and perpendicular to the layer plane. 


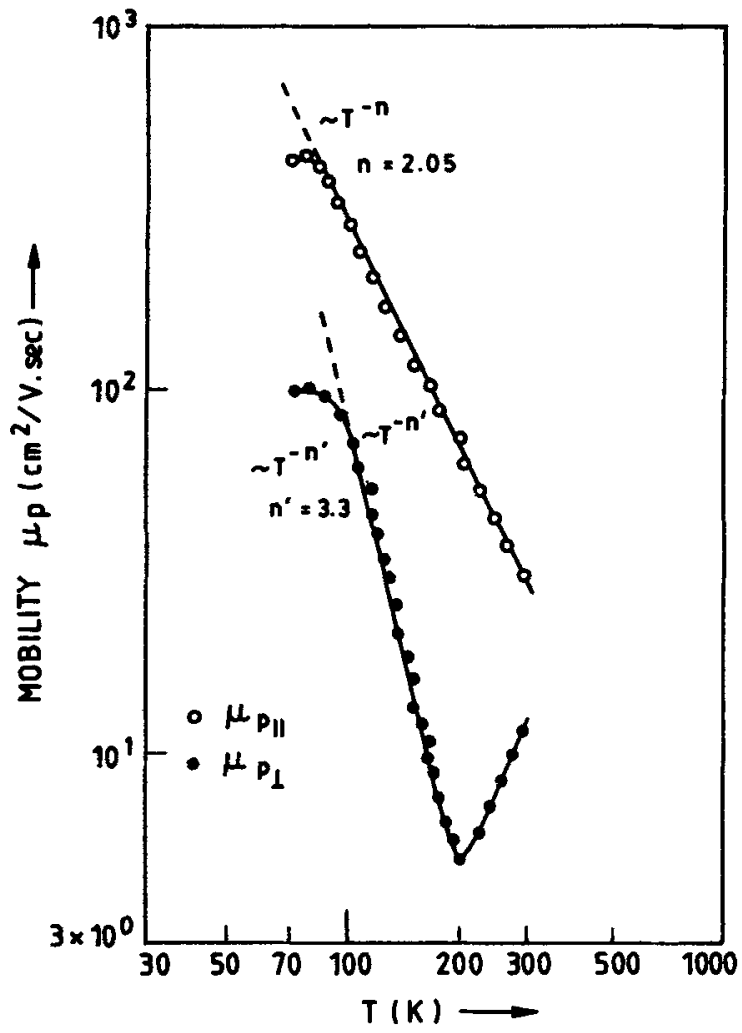

Figure 3. Variation of hole mobility with temperature in two directions $(\log \mu$ vs $\log T)$.

higher than the highest mobility of $\mu_{11}=150 \mathrm{~cm}^{2} / V \mathrm{sec}$ reported by Augelli et al (1977) attesting to the high quality of the present crystals. The hole mobility had a temperature dependence for $T>100 \mathrm{~K}$ given by $T^{-n}$ where $n=2.05$ parallel to and 3.3 perpendicular to the layer plane.

The temperature dependence of $\mu_{11}$ has been explained by Fivaz and Schmid (1976) as due to scattering by homopolar optical phonons polarized parallel to the layer planes. Good agreement with this theory was obtained assuming interaction with an optical phonon of energy $\hbar \omega=0.014 \mathrm{eV}$ which was observed in Raman studies by Cerdeira et al (1977). If the carrier-lattice coupling constant $g^{2}=0.31$ as estimated by Camassel et al (1977), the in-plane effective mass $m *=0.47 m_{0}$ is in excellent agreement with TEP results. The hole density-of-states effective mass was estimated by Manfredotti et al (1975) to be $0.6 \mathrm{~m}_{0}$. From this the effective mass perpendicular to the layer planes is found to be $1.0 \mathrm{~m}_{0}$, again in good agreement with the value of $0.995 m_{0}$ obtained from TEP (table 2).

The Hall mobility $\mu_{\perp}$ showed a minimum with a thermally activated behaviour for $T>200 \mathrm{~K}$ with $\Delta E=0.040 \mathrm{eV}$ as observed in the case of the conductivity anisotropy. Similar behaviour has been observed in GaSe and is attributed to the presence of interlayer stacking faults. 


\section{2D localization studies}

Weak localization in InSe has been reported by El-Khatouri et al (1989) who examined conductivity variation with temperature but only along the layer planes. Conductivity studies were thus carried out for GaTe between $10-50 \mathrm{~K}$ in a closed cycle $\mathrm{He}$ cryostat with the electric field parallel or perpendicular to the layer planes. Results are given in figures $4 a$ and $b$ and summed up in table 3.

It is seen (figure 4a) that parallel to the layer plane weak localization occurs for $T<20 \mathrm{~K}$ the relation followed being given by (Lee and Ramakrishnan 1985),

$$
\Delta G=\left(\lambda e^{2} / \hbar^{2}\right) \ln T
$$

where $\lambda=\alpha p^{2} / 2 \pi^{2}$. From experiment $\lambda=0.051$ giving $p=1$ for a non-interacting electron gas with $\alpha=1$. The value of $\left(e^{2} / 2 \pi^{2} h\right)$ was found from the slope to be $1.23 \times 10^{-5}$ mhos.
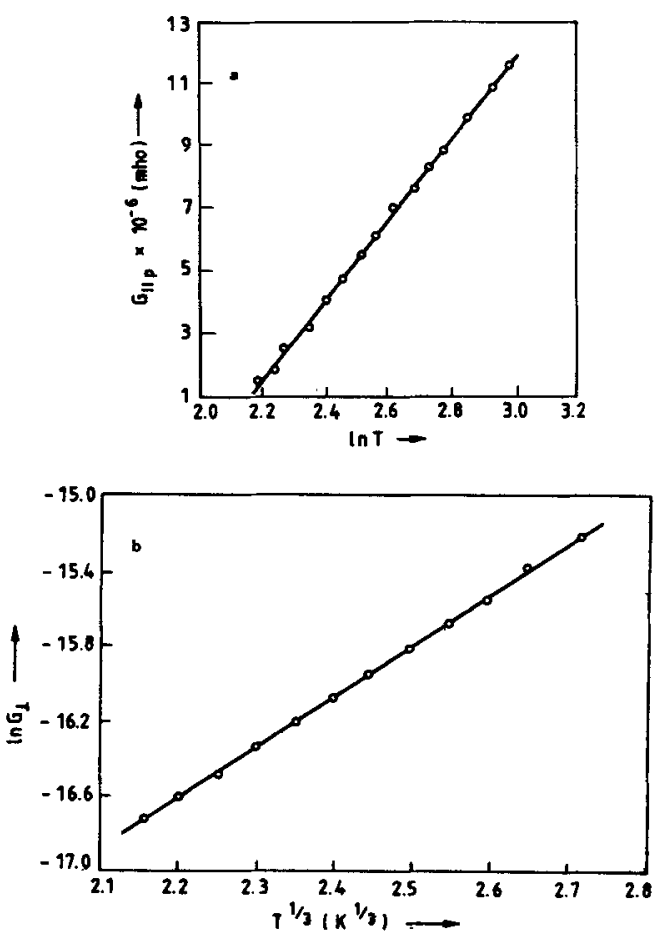

Figure 4. a. Conductance $G$ vs $\ln T$ along the layer planes for $T=9-20 \mathrm{~K}$ and b. conductance ln $G$ vs $T^{1 / 3}$ perpendicular to the layer planes for $T=9-20 \mathrm{~K}$.

Table 3. Conductivity variation of GaTe in orthogonal directions.

\begin{tabular}{lcc}
\hline Direction & $T=9-20 \mathrm{~K}$ & $T=20-50 \mathrm{~K}$ \\
\hline Parallel to layer plane & $G \propto \ln T$ & $\sigma \propto T^{1 / 2}$ \\
Perpendicular to plane & $G \propto \exp \left(T / T_{0}\right)^{1 / 3}$ & $\sigma \propto \exp \left(T / T_{0}\right)^{1 / 4}$ \\
\hline
\end{tabular}


For $T=20-50 \mathrm{~K}$ the $T^{1 / 2}$ variation is a consequence of 3-dimensional conduction under weak localization which is proportional to $T^{p / 2}$, where $p=1$ in the present case.

In the perpendicular direction the temperature variation (figure $4 \mathrm{~b}$ ) is characteristic of 2-D hopping for $T<20 \mathrm{~K}$ with dimensional crossover to 3-D Mott-type hopping for $T>20 \mathrm{~K}$. These results have been discussed in detail by Basak (1994).

The two-dimensional nature of conduction parallel to the layer planes resulted in negative longitudinal magnetoresistance (positive magnetoconductance) being observed at low temperatures with relatively weak fields $<0.4 \mathrm{~T}$. The results are depicted in figure $5 \mathrm{a}$ and summarized in table 4 . At $10 \mathrm{~K}$ the application of magnetic field results in suppression of weak localization and hence increase in conductance which is proportional to $\mathrm{H}^{2}$ (figure $5 \mathrm{~b}$ ). At $T=80 \mathrm{~K}$ for band conduction, increase in scattering and decrease in hole mean-free-path due to the applied magnetic field results in positive magnetoresistance as expected.
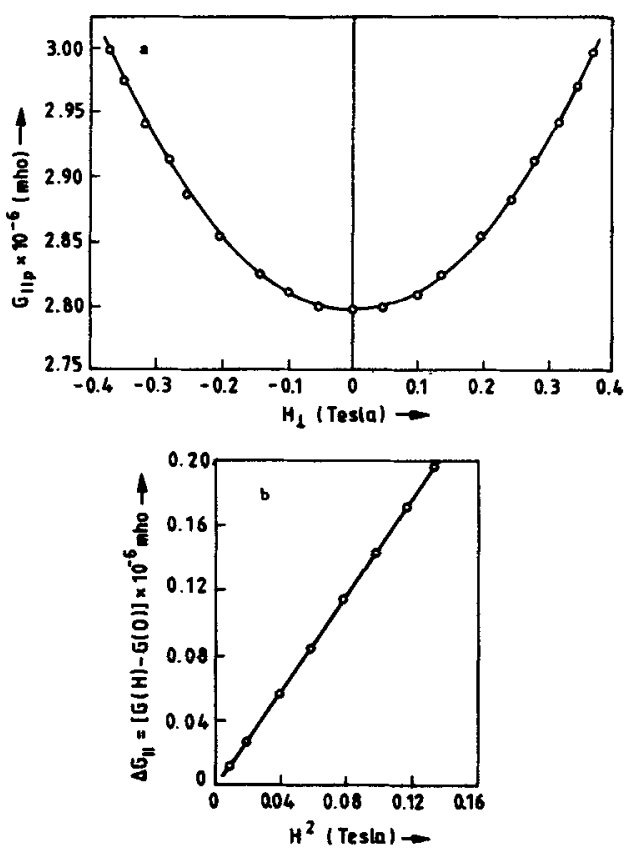

Figure 5. a. Transverse magnetoconductance in the layer plane at $T=10 \mathrm{~K}$ and b. change in conductance $\Delta G$ vs $H^{2}$ at $T=10 \mathrm{~K}$.

Table 4. Magnetoresistance in GaTe.

\begin{tabular}{llc}
\hline \multirow{2}{*}{ Direction } & \multicolumn{2}{c}{$(\Delta \rho / \rho)(\%)$} \\
\cline { 2 - 3 } & $T=10 \mathrm{~K}$ & $T=80 \mathrm{~K}$ \\
\hline Parallel to layers & $-7.14 \%$ & $+11.5 \%$ \\
Perpendicular to layers & $-5.18 \%$ & $+8.3 \%$ \\
\hline
\end{tabular}




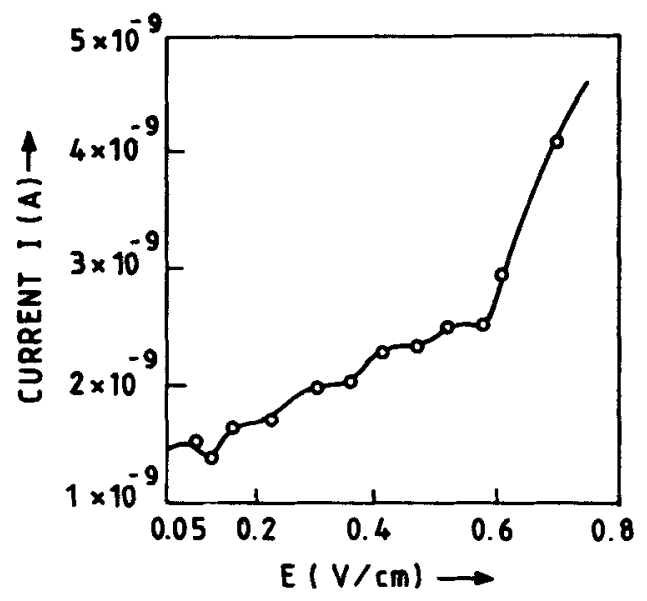

Figure 6. $I-V$ characteristic across the layer plane at $T=10 \mathrm{~K}$.

\section{I-V characteristics}

The I-V characteristics of the samples were studied at $10-25 \mathrm{~K}$ at low electric fields both in and across the layer planes. In the latter case it was found that $I$ showed an initial decrease indicating negative differential resistance (NDR) and then increased in discrete steps at $10 \mathrm{~K}$ (figure 6), the effect vanishing at $25 \mathrm{~K}$. This behaviour is similar to the resonant tunneling (RT) observed in MBE grown $\mathrm{GaAs} / \mathrm{Al}_{x} \mathrm{Ga}_{1-x}$ As superlattices at $77 \mathrm{~K}$ and $300 \mathrm{~K}$ by Morkoc et al (1986) which also showed NDR. Resonant tunneling of holes, as in the present case, was observed in AlAs/GaAs quantum well structures by Mendez et al (1985). RT is due to the applied bias bringing successive quasi-bound states in the narrow regions confined by the potential barriers into alignment permitting tunneling and resulting in the observed current steps. At $T>20 \mathrm{~K}$ thermal excitation over the potential barriers becomes possible while at higher fields field-assisted tunneling takes over. In the GaAs/AlAs system the confining potential for holes is $\sim 150 \mathrm{meV}$ and well widths are typically less than $10 \mathrm{~nm}$ which gives large separation between the quantized levels. Hence NDR can be observed at $300 \mathrm{~K}$. For GaTe the confining potentials due to stacking faults are estimated to be a few meV and potential wells are much wider so that the phenomenon is only observed at $T<10 \mathrm{~K}$. This is to the authors knowledge the first report of such an observation in a natural superlattice.

\section{Conclusion}

The study of anisotropy of the transport properties of the layered chalcogenide GaTe revealed novel phenomena which can be explained on the basis of weak localization at low temperatures and its removal due to applied magnetic field resulting in negative magnetoresistance. The anisotropy of effective mass in orthogonal directions was determined from TEP studies while the temperature dependence of mobility $\mu_{1 \mid}$ indicated homopolar optical scattering. Resonant tunneling with NDR was observed in such a natural superlattice for the first time. 


\section{Acknowledgement}

One of the authors (SP) is grateful to the University Grants Commission for the award of a Fellowship.

\section{References}

Augelli V, Manfredotti C, Murri R, Piccolo R, Rizzo A and Vasanelli L 1977 Solid State Commun. 21 575

Basak S 1994 Growth, characterization, electrical and dielectric properties of layered chalcugenides InTe-GaTe, Ph.D. Dissertation, IIT, Kharagpur

Bose D N and Pal S 1994 Mater. Res. Bull. 29 2, 111

Brebner J L and Fischer G 1962 Proc. int. conf. phys. semiconductors, Exeter, (London: Inst. of Phys. \& Phys. Soc.) p. 760

Camassel J, Merle P, Mathieu H and Gouskov A 1977 Phys. Rev. B19 1060

Cerdeira E, Meneses E A and Gouskov A 1977 Phys. Rev. B19 1648

El-Khatouri D, Khater A, Balkanski M. Julien M and Guesdon J P 1989 J. Appl. Phys. 662049

Fischer G and Brebner J L 1962 J. Phys. Chem. Solids 231363

Fivaz R C and Schmid P E 1976 Physics and chemistry of materials with layered structures (Dordrecht: Reidel) Vol. 4

Lee P A and Ramakrishnan T V 1985 Rev. Mod. Phys. 57287

Manfredotti C, Murri R, Rizzo A, Vasanelli L and Micocci G 1975 Phys. Status Solidi B65 249

Mendez A A, Wang W I, Ricco B and Esaki L 1985 Appl. Phys. Lett. 47415

Morkoc H, Chen J, Reddy U K, Henderson T and Luryi S 1986 Appl. Phys. Lett. 4970

Pal D. Pal S and Bose D N 1994 Bull. Mater. Sci. 17347

Segura A, Martinez-Tomas C, Casanovas A, Cantareo A, Martinez-Pastor A and Chevy A 1989 Appl. Phys. A45 445 